\title{
Effects of $B C L 2$ transfection on the cell cycle and proliferation of human GES-1 cells
}

\author{
G.Y. Shi ${ }^{1}$, L.L. Zhao' ${ }^{2}$ K. Zhang ${ }^{3}$, H.X. Zhou ${ }^{4}$, A.H. Liu' ${ }^{5}$ J. Li $^{5}$, \\ G. $\mathrm{Li}^{6}$ and L.H. $\mathrm{Zhu}^{5}$ \\ ${ }^{1}$ Jitang College, North China University of Science and Technology, \\ Tangshan, China \\ ${ }^{2}$ Pharmacy Disciplines, Pharmaceutical College, \\ North China University of Science and Technology, Tangshan, China \\ ${ }^{3}$ College of Adult Education, \\ North China University of Science and Technology, Tangshan, China \\ ${ }^{4}$ Department of Anatomy, School of Basic Medical, \\ North China University of Science and Technology, Tangshan, China \\ ${ }^{5}$ Department of Pathogeny Biology, School of Basic Medical, \\ North China University of Science and Technology, Tangshan, China \\ ${ }^{6}$ Affiliated Hospital, North China University of Science and Technology, \\ Tangshan, China \\ Corresponding author: L.H. Zhu \\ E-mail: zlhphd@163.com
}

Genet. Mol. Res. 14 (4): 12022-12029 (2015)

Received January 28, 2015

Accepted June 8, 2015

Published October 5, 2015

DOI http://dx.doi.org/10.4238/2015.October.5.15

\begin{abstract}
We investigated the effects of $B C L 2$ transfection on the cell cycle and proliferation of GES-1 cells. A pcDNA3-BCL2 plasmid was used to transfect GES-1 cell line human gastric epithelial cells. Clones were obtained by G418 screening. BCL2-positive cells were identified by fluorescence immunohistochemistry. The pcDNA3-BCL2 vectors carrying the $N e o R$ gene were transfected into GES-1 cells, while the empty plasmid was transfected into the same cells as controls. BCL2-positive clones were screened by neomycin 418 (G418). Flow
\end{abstract}


cytometry was used to detect the cell cycle. Hematoxylin and eosin (H\&E) staining revealed morphological changes, and the effects of $B C L 2$ transfection on cell proliferation were analyzed by cell counting and 3-(4,5-dimethylthiazol-2-yl)-2,5-diphenyltetrazolium bromide assay. The plasmid pcDNA3-BCL2 was identified by restriction enzyme digestion. Different degrees of $B C L 2$ gene expression were detected in all seven clones. BCL2 was expressed mainly in the cytoplasm and the nuclear membrane. There were significantly more S-phase cells in the transfection group than in the controls. The morphology did not change after $\mathrm{H} \& \mathrm{E}$ staining. Cell growth was faster than in the controls after transfection for 6 days. At 24, 48, and $72 \mathrm{~h}$ after transfection, the A values were $4.15 \pm 0.31,5.98 \pm 0.56$, and $8.94 \pm 0.79$; those of the controls were $3.01 \pm 0.20,4.76 \pm 0.52$, and $7.69 \pm 0.84$; there was a significant difference between the two groups $(\mathrm{P}<0.05) . B C L 2$ transfection increased GES-1 cells in the S phase; the GES-1 cells were stable and BCL2 expression was high, which promoted cell proliferation.

Key words: $B C L 2$ gene; Human gastric epithelial cells; GES-1 cells; Transfection; Cell cycle

\section{INTRODUCTION}

Although gastric carcinoma (GC) is a common malignancy of the digestive system, its carcinogenic mechanism is not very clear. Most researchers believe that GC incidence depends on a multi-factorial (including physical, chemical, and biological factors) and multi-stage process. GC involves high expression levels of multiple oncogenes (Rigoli and Caruso, 2014). BCL2 is the shortened form used for the B-cell lymphoma/ leukemia-2 gene. It was the first gene to be confirmed as apoptosis inhibiting (Xu et al., 2014), and has the chromosomal locus 18q21. Abnormal expression of BCL2 is related to a variety of diseases, and is particularly closely related to the incidence and development of cancers. The BCL2 protein is located in the mitochondrial membrane, the smooth endoplasmic reticulum, and around the nuclear membrane in cells, and can be expressed in the activation process of various normal tissues and cells. High expression of the $B C L 2$ gene can inhibit apoptosis induced by a variety of factors (Solórzano et al., 2005), and may be directly involved in cell proliferation and survival. Experiments on transgenic animals and gene transfection have shown that the $B C L 2$ gene can significantly inhibit cell death (Kawatani and Imoto, 2003).

Vaux et al. (1988) were the first to confirm that $B C L 2$ can inhibit apoptosis to prolong cell death. A previous study (de la Coste et al., 1999) had suggested that abnormally high expression of the BCL2 protein in $B c l 2$ transgenic mice led to the prolonged survival of $\mathrm{B}$ cells. These experiments strongly confirm the delaying role of the $B C L 2$ gene in cell death. It is now generally agreed that despite the inhibition of cell apoptosis by the $B C L 2$ gene, the gene itself does not lead to tumorigenesis; it merely prolongs cell survival, thereby providing opportunities for mutations in cell proliferation or cell proliferation-inhibiting genes, resulting 
in tumorigenesis. Abnormal expression of the $B C L 2$ gene has been found not only in lymphohematopoietic malignancies, but also in non-lymphohematopoietic tumors, such as nasopharyngeal carcinoma, $\mathrm{GC}$, and lung cancer. In this study, the $B C L 2$ oncogene was transfected into normal gastric epithelial cells to analyze the effects of the $B C L 2$ gene on the cell cycle and the proliferation of gastric epithelial cells.

\section{MATERIAL AND METHODS}

\section{Reagents}

Lipofectamine, Dulbecco's modified Eagle's medium (DMEM), and Roswell Park Memorial Institute 1640 (RPMI 1640) culture medium were purchased from the Gibco Brl Company (USA); fetal bovine serum (FBS) was purchased from the Tianjin Biochemical Products Factory; mouse anti-BCL2 IgG and fluorescein isothiocyanate (FITC)-labeled goat anti-mouse IgG were purchased from the Beijing Huamei Biological Company; other chemical reagents were of analytical grade. The plasmid pcDNA3-BCL2 was donated by Professor Kenzo Takada, Department of Oncology Virology, Institute of Genetic Medicine, Medical College of Hokkaido University.

\section{Cell line}

Normal human gastric epithelial cell line GES-1 was purchased from the Beijing Institute of Cancer Research.

\section{Extraction and identification of plasmid pcDNA3-BCL2}

Miniprep plasmid pcDNA3-BCL2 was extracted using the alkaline lysis method, and the $B C L 2$ gene for transfection was identified after EcoRI digestion by agarose gel electrophoresis.

\section{Cell transfection}

Cell transfections were conducted according to the Lipofectamine manufacturer instructions (You et al., 2014; Aoyama et al., 2015). Briefly, the steps were as follows: GES-1 cells in the logarithmic growth phase were seeded on 24-well plates, each well containing 0.5 mL RPMI 1640 medium with $10 \%$ FBS; cells were incubated at $37^{\circ} \mathrm{C}$ in $5 \% \mathrm{CO}_{2}$ until $70 \%$ confluence, then the cell culture was discarded and the cells were washed once with serumfree DMEM. The pcDNA3-BCL2 vector $(0.4 \mu \mathrm{g})$ was dissolved in $50 \mu \mathrm{L}$ serum-free DMEM as solution $\mathrm{A} ; 1 \mu \mathrm{L}$ Lipofectamine was added to $49 \mu \mathrm{L}$ serum-free DMEM as solution B. Solutions A and B were gently mixed and kept at room temperature for 20 min to obtain the DNAliposome complex. Serum- and antibiotic-free DMEM $(500 \mu \mathrm{L})$ was then added. The solution was mixed gently and added to the washed cells, which were incubated at $37^{\circ} \mathrm{C}$ in $5 \% \mathrm{CO}_{2}$ for $8 \mathrm{~h}$. The transfection solution was discarded and $0.5 \mathrm{~mL}$ RPMI 1640 medium containing $10 \% \mathrm{FBS}$ was added for further culture. After gene transfection for $48 \mathrm{~h}$, the complete medium containing $300 \mu \mathrm{g} / \mathrm{mL}$ Geneticin 418 (G418) was added for screening. 


\section{Flow cytometry to detect the cell cycle}

The stably transfected cells were digested with $0.25 \%$ trypsin, then collected by centrifugation and washed twice with cold phosphate-buffered saline (PBS). Pre-cooled 70\% ethanol was added for fixing at $4^{\circ} \mathrm{C}$ overnight. After centrifugation at $800-1000 \mathrm{~g}$, the supernatant was removed. The cells were centrifuged and washed twice with cold PBS, then resuspended to make a single cell suspension; the centrifugation speed should not be excessive. The cell suspension and propidium iodide dye were mixed in equal volumes, and left to stand at $4^{\circ} \mathrm{C}$ for $30 \mathrm{~min}$. After filtering on a 300 mesh nylon filter, the sample was placed in the sample chamber of a flow cytometer for cell cycle detection.

\section{Detection of $B C L 2$ expression by FITC-labeled immunohistochemistry}

The creep plates of transfected cells and normal GES-1 cells were collected and fixed. Mouse anti-BCL2IgG (1:50) was placed in a wet box overnight at $37^{\circ} \mathrm{C}$. After washing with PBS, FITC-labeled goat anti-mouse IgG (1:50) was incubated at $37^{\circ} \mathrm{C}$ for $30 \mathrm{~min}$. After PBS washing, glycerin/PBS (9:1) was used for sheet sealing. Under a fluorescence microscope, the positive part was yellow-green; depending on the intensity of coloration, it was divided into positive and negative; the observation and photography were performed within 5-15 min.

\section{Cell growth detection}

The cells were seeded on 24-well plates at a density of $3 \times 10^{6} / \mathrm{L}$ each well. Each day, three wells were selected for cell counting for 10 days to produce a growth curve, measuring the total number of cells in each well and calculating the average for each group. Cells were then seeded on 96-well plates. After culture for 24 , 48, and $72 \mathrm{~h}, 20 \mu \mathrm{L} 3$-(4,5-dimethylthiazol-2-yl)-2,5-diphenyltetrazolium bromide was added to each well $(5 \mathrm{~g} / \mathrm{L})$. Incubation was continued for $4 \mathrm{~h}$ at $37^{\circ} \mathrm{C}$, then cell culture was terminated and the culture supernatant was carefully aspirated in each well. Dimethyl sulfoxide $(150 \mu \mathrm{L})$ was added to each well and oscillation was carried out for 10 min to fully dissolve the crystals. The absorbance value at $490 \mathrm{~nm}$ of each well was measured in the enzyme-linked immunoassay meter and the results were recorded.

\section{Statistical analysis}

Experimental results are reported as mean \pm SD. The SPSS 11.5 software package was used for statistical analysis. Comparison between groups was analyzed using one-way analysis of variance. Pairwise comparison was analyzed using the Dunnett $t$-test.

\section{RESULTS}

\section{Results of plasmid pcDNA3-BCL2 extraction and identification}

The $B C L 2$ gene was located at $900 \mathrm{bp}$ on the electrophoresis map (Figure 1), indicating that transfected plasmids carried the $B C L 2$ gene. 


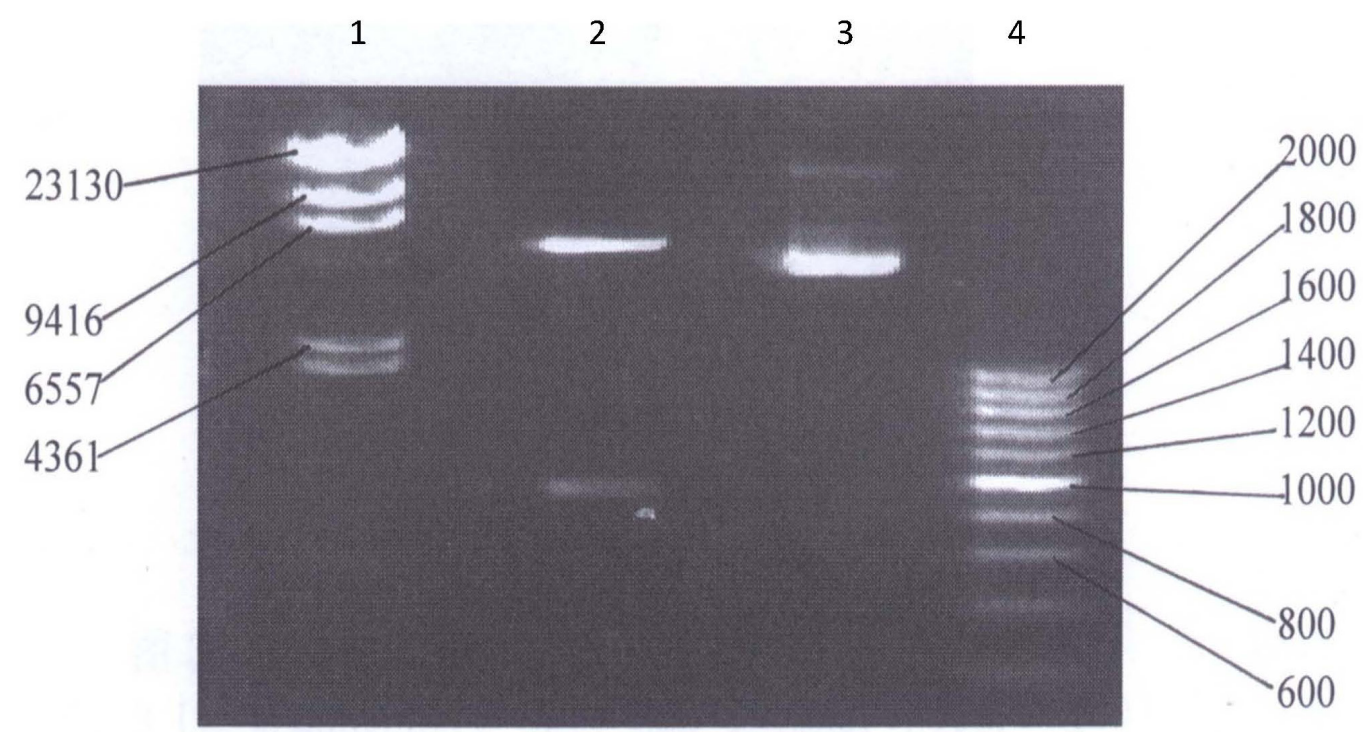

Figure 1. Identification of pcDNA3-BCL2 by restriction endonuclease digestion. Lane $1=\mathrm{DNA} /$ HindIII marker; lane 2 = pcDNA3-BCL2 digestion by EcoRI; lane 3 = pcDNA3-BCL2; lane $4=200$-bp DNA step ladder.

\section{Results of cell transfection and screening}

To establish a stable expression system in the human gastric epithelial GES-1 cell line transfected with $B C L 2$, transfected cells were screened with G418. The results showed that after transfection and G418 screening, all the cells in the untransfected group were dead. In contrast, the formation of transfected cells was clear and a large number of cell colonies existed, indicating effective transfection and screening (Figure 2A).
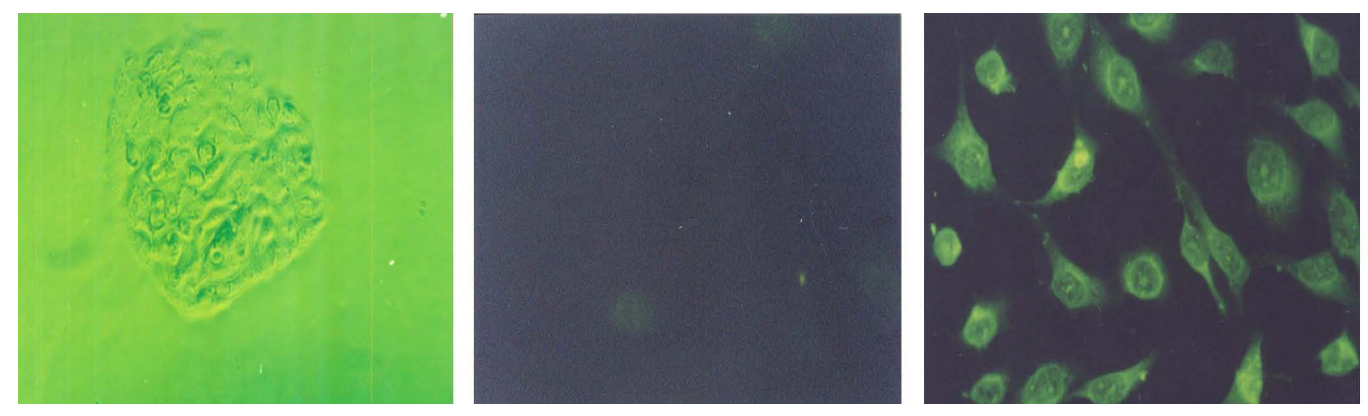

Figure 2. GES-1 cell clones in the different groups. A. BCL2 gene-transfected GES-1 cell clones (200X magnification); B. Untransfected GES-1 cells [by immunohistochemistry (IHC) using fluorescein isothiocyanate (FITC) at 400X magnification]; C. BCL2-transfected GES-1 cells (IHC, FITC, 400X).

\section{Identification of the expression of BCL2}

After screening, seven screened colonies were randomly selected for immuno- 
fluorescence staining to identify the expression of BCL2. The results showed that in the seven transfected GES-1 cell colonies, six highly expressed BCL2 and only one had relatively weak expression. The cells in the untransfected group showed no BCL2 expression (Figure 2B and C).

\section{Detection of cell cycle}

As shown in Figure 3A-C, in the cycle maps of normal GES-1 cells, the cell distributions in the G1, G2, and S phases accounted for $34.42,16.23$, and $49.35 \%$, respectively. In the cell cycle maps of GES-1 cells transfected with empty vectors, the cell cycle distributions in the G1, G2, and S phases accounted for 39.63, 25.63, and 34.74\%, respectively. In the cell cycle maps of GES-1 cells transfected with the BCL2 gene, the distributions in the G1, G2, and S phases accounted for $33.44,4.09$, and $62.47 \%$, respectively. Comparing the distributions of cells at each phase, we can conclude that BCL2-transfected cells had an extended S phase (Table 1).

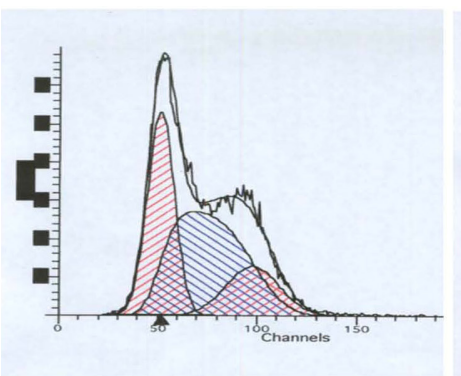

A

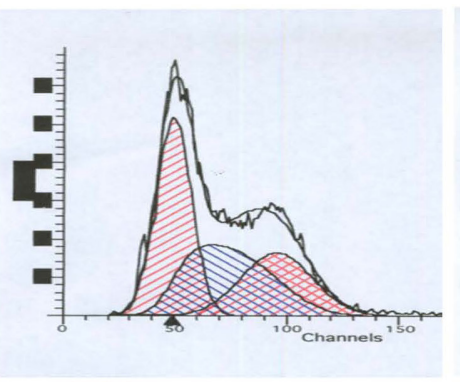

B

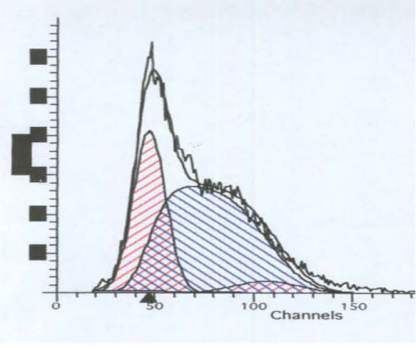

$\mathrm{C}$

Figure 3. GES-1 cell cycle in the different groups. A. Normal; B. empty plasmid transfection; C. BCL2 gene transfection.

Table 1. Flow cytometry analysis for the effect of $B C L 2$ gene transfection on the cell cycle.

\begin{tabular}{lcrr}
\hline Groups & \multicolumn{3}{c}{ Proportion of cells in each phase (\%) } \\
\cline { 2 - 4 } & $\mathrm{G} 1$ & $\mathrm{~S}$ & $\mathrm{G} 2 / \mathrm{M}$ \\
\hline Normal & $34.42 \pm 3.19$ & $49.35 \pm 1.16$ & $16.23 \pm 1.18$ \\
Empty plasmid transfection & $39.63 \pm 3.81$ & $34.74 \pm 1.03$ & $25.63 \pm 2.06$ \\
BCL2 gene transfection & $33.44 \pm 4.31^{*}$ & $62.47 \pm 2.88^{*}$ & $4.09 \pm 0.85^{*}$ \\
\hline
\end{tabular}

$* \mathrm{P}<0.05$.

\section{Cell growth}

From the growth curve we can conclude that the growth rate of $B C L 2$-transfected cells was significantly higher after 4 days (Figure 4). After culture for 24, 48, and 72 $\mathrm{h}$, the proliferation of transfected cells increased significantly. After statistical analysis, there were significant differences $(\mathrm{P}<0.05$, Table 2$)$ between the transfection group and the control group. 


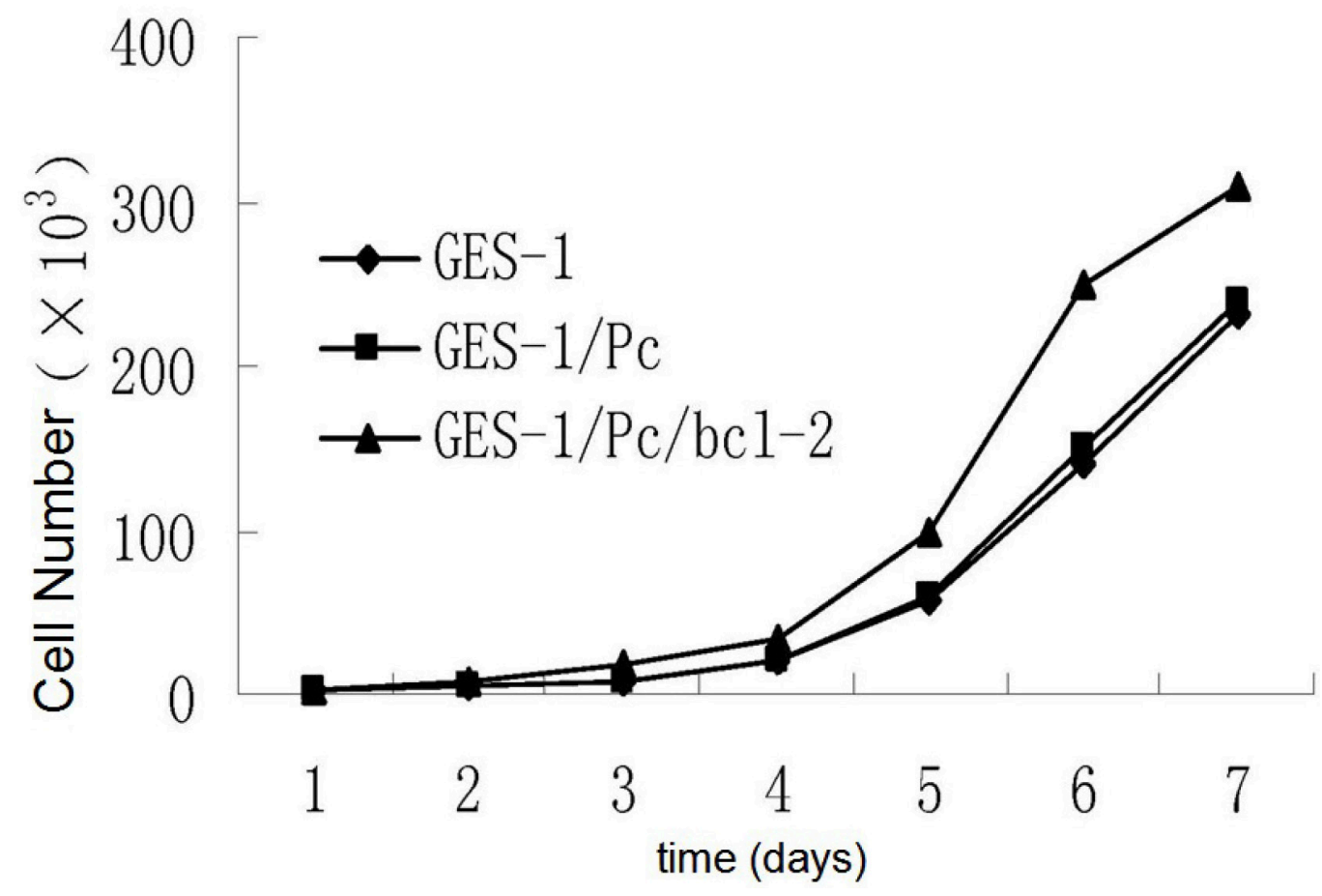

Figure 4. Growth curve of GES-1 cells.

Table 2. Cell proliferation after transfection with the $B C L 2$ gene $(\mathrm{N}=4)$.

\begin{tabular}{lccc}
\hline Cell & $24 \mathrm{~h}$ & $48 \mathrm{~h}$ & $72 \mathrm{~h}$ \\
\hline GES-1 & $2.93 \pm 0.22$ & $4.54 \pm 0.35$ & $7.15 \pm 0.67$ \\
PcDNA & $3.01 \pm 0.20$ & $4.76 \pm 0.52$ & $7.69 \pm 0.84$ \\
PcDNA-BCL2 & $4.15 \pm 0.31^{\mathrm{a}}$ & $5.98 \pm 0.56^{\mathrm{a}}$ & $8.94 \pm 0.79^{\mathrm{a}}$ \\
\hline
\end{tabular}

${ }^{a} \mathrm{P}<0.05$.

\section{DISCUSSION}

$B C L 2$ is a recently discovered cell apoptosis gene. Its abnormal expression is correlated with the development and prognosis of a variety of diseases, especially cancer. Vaux et al. (1988) first confirmed that $B C L 2$ can inhibit apoptosis and prolong cell death. Domestic studies have reported that the rate of BCL2 protein expression in GC tissues is $60-70 \%$. The expression of BCL2 occurs in early GC, because it functions at the start-up phase of GC. This study established a $B C L 2$ gene-transfected GES-1 human gastric epithelial cell model, which can be used for in vitro cell culture to further study the synergy of the $B C L 2$ gene with other genes (such as C-MYC) and other factors (such as viral infection) in gastric carcinogenesis.

High expression of the BCL2 gene can inhibit apoptosis caused by a variety of apoptosis-inducing factors, and may be directly involved in cell proliferation and cell survival. 
Through experiments on transgenic animals and gene transfection, it was found that the BCL2 gene can significantly inhibit cell death. This study showed that after transfection with the $B C L 2$ gene, the S phase of the transfected cells was extended, which lays the foundation for further studies on the exact role and action mechanism of the $B C L 2$ gene in the incidence and development of GC.

As mentioned above, the high expression of $B C L 2$ protected against a variety of apoptotic stimuli. Therefore, low expression or absence of $B C L 2$ led to a significant increase in cell death. The $B C L 2$ gene has been proven to play an important role in the inhibition of apoptosis in transformed cells, which can resist and suppress apoptosis induced by a variety of factors, enhance cell viability, and participate in the regulation of cell proliferation and apoptosis. $B C L 2$ transfection can inhibit cell apoptosis induced by chemotherapy drugs, heating, radiation, cell growth factor withdrawal, c-myc, and p53 to prolong cell survival. Using liposomemediated gene transfection, we transferred the eukaryotic expression vector pcDNA3 containing human BCL2 complementary DNA into human gastric epithelial cell line GES-1, and successfully established a GES-1-BCL2 clone with 100\% stable expression of BCL2 protein.

\section{Conflicts of interest}

The authors declare no conflict of interest.

\section{ACKNOWLEDGMENTS}

Research supported by the Science and Technology of Hebei Province, China (\#132777138).

\section{REFERENCES}

Aoyama K, Yamaguchi N, Yuki R, Morii M, et al. (2015). c-Abl induces stabilization of histone deacetylase 1 (HDAC1) in a kinase activity-dependent manner. Cell Biol. Int. 39: 446-456.

de la Coste A, Fabre M, McDonell N, Porteu A, et al. (1999). Differential protective effects of Bcl-xL and Bcl-2 on apoptotic liver injury in transgenic mice. Am. J. Physiol. 277: G702-G708.

Kawatani M and Imoto M (2003). Deletion of the BH1 domain of Bcl-2 accelerates apoptosis by acting in a dominant negative fashion. J. Biol. Chem. 278: 19732-19742.

Rigoli L and Caruso RA (2014). Mitochondrial DNA alterations in the progression of gastric carcinomas: unexplored issues and future research needs. World J. Gastroenterol. 20: 16159-16166.

Solórzano L, Rieber MS, Medina JD and Rieber M (2005). Decreased glycolytic metabolism accelerates apoptosis in response to 2-acetyl furanonaphthoquinone in K1735 melanoma irrespective of bcl-2 overexpression. Cancer Biol. Ther. 4: 329-335.

Vaux DL, Cory S and Adams JM (1988). Bcl-2 gene promotes haemopoietic cell survival and cooperates with c-myc to immortalize pre-B cells. Nature 335: 440-442.

Xu L, Jin QD, Gong X, Liu H, et al. (2014). Anti-gastric cancer effect of melatonin and Bcl-2, Bax, p21 and p53 expression changes. Sheng Li Xue Bao 66: 723-729.

You MK, Lim SH, Kim MJ, Jeong YS, et al. (2014). Improvement of the fluorescence intensity during a flow cytometric analysis for rice protoplasts by localization of a green fluorescent protein into chloroplasts. Int. J. Mol. Sci. 16: 788804. 